\title{
Sweet and sour rats: The effect of insulin dosage on shock-elicited aggression
}

\author{
JERRY NEIDEFFER \\ Harriet Cohn Mental Health Center, Clarksville, Tennessee 37040 \\ MARY NELL TRAVIS, STEPHEN F. DAVIS, and JAMES W. VOORHEES \\ Austin Peay State University, Clarksville, Tennessee 37040 \\ and \\ ROBERT E. PRYTULA \\ Middle Tennessee State University, Murfreesboro, Tennessee 37132
}

The present study sought to determine what, if any, relationship exists between blood-sugar level and aggression. Three groups of rats had blood-sugar level experimentally reduced via injections of 4,8 , and 12 units of regular zinc insulin. A fourth group served as an injection control. One-half hour following its injection, each subject received $10 \mathrm{~min}$ of shock-elicited aggression testing. The results indicated that the subjects receiving the 12 -unit insulin injection were significantly more aggressive than all other groups. Graphically there was an indication that the larger the insulin dosage (i.e., the lower the blood-sugar level), the higher the amount of aggression displayed.

Hypoglycemia can be casually characterized as a condition involving low levels of blood sugar. Conversations with hypoglycemics have suggested, rather uniformly, that a subjective state of irritation and possibly increased aggressiveness accompanies and may be produced by this lowered level of blood sugar. Unfortunately, these reports linking aggression and lowered blood-sugar level are quite speculative and have little, or no, experimental basis. However, some support is provided by Trotter (1973), who studied the Qolla, an Indian subculture living in Latin America. Trotter (1973) characterized their behavior as mean, hostile, and aggressive, with violent encounters being the rule rather than the exception. In trying to establish the basis for this behavior, Trotter found that 55\% of the Qolla who underwent a 4-h glucose tolerance test were suffering from mild to severe hypoglycemia. This certainly lends some support, although indirect, to the proposition that lowered blood-sugar level was one of the causes, if not the cause, of the extreme incidence of aggressiveness observed in this group.

To date it would appear that no attempt has been made to experimentally link level of blood sugar and amount of aggression. The present study sought to investigate this proposed functional relationship.

This research was supported by a Tower Fund research grant from Austin Peay State University to the third author and by a faculty research grant from Middle Tennessee State University to the last author. Requests for reprints should be directed to Stephen F. Davis, Department of Psychology, Austin Peay State University, Clarksville, Tennessee 37040.

\section{METHOD}

\section{Subjects}

Forty-eight 90-day-old male albino rats purchased from the Sprague-Dawley Company, Madison, Wisconsin, served as subjects. All subjects were individually caged with food and water continuously available.

\section{Apparatus}

All subjects were tested in a shock-elicited aggression apparatus consisting of an opaque restraint tube, shock source, aggression target, and recording device (see Mollenhour, Voorhees, \& Davis, 1977, for a more complete description). One end of the restraint tube was open and faced an omnidirectional lever which served as the aggression target. Attack upon this target in turn activated an impulse counter, thus allowing the experimenter to keep an automated record of the number of aggressive responses. A small hole in the other end of the tube allowed the subject's tail to be extended from the tube. Once the tail was extended from the tube, a wooden rod was secured in place by means of adhesive tape, thus prohibiting escape during shock testing. Two copper wires attached to the wooden rod served as electrodes for the administration of shock.

\section{Procedure}

At the beginning of the experiment four equal groups were randomly formed. Three of the groups (Groups 4, 8, and 12) had blood-sugar level experimentally reduced via insulin injection. The fourth group (Group C) received an injection of $.06 \%$ phenol and served as an injection-control group. Insulin doses were 4,8 , and 12 units of $U 40$ zinc insulin, and were conceptually thought of as reflecting low, moderate, and high reductions in blood-sugar level, respectively. All groups received a 12-unit injection to control for amount of substance injected. Thus, subjects in the 4-unit group received 4 units of insulin plus 8 units of phenol, subjects in the 8-unit group received 8 units of insulin plus 4 units of phenol, and subjects in the control group received 12 units of phenol. All injections were administered $1 / 2 \mathrm{~h}$ prior to testing. The injection schedule was staggered 
so that each subject would have an individual testing session. The order for injecting, and hence the order for running subjects, was randomized.

A test session was conducted by removing the test subject from the home cage $1 / 2 \mathrm{~h}$ after the designated injection. The subject was secured in the restraint tube and allowed $5 \mathrm{~min}$ to habituate. At the end of the 5-min habituation period, a 10-min shock period was begun. During this time each subject received 2001.5 -mA (half-wave DC) shocks of 300-msec duration administered at 3-sec intervals.

\section{RESULTS AND DISCUSSION}

Mean aggressive responses for the four groups are shown in Figure 1. Analysis of variance yielded a significant groups effect $[F(3,44)=6.00, p<.01]$. Additional Newman-Keuls indicated that Group 12 was significantly more aggressive $(p<.05)$ than all other groups and that these groups, in turn, did not differ among themselves.

Obviously, these results lend support to our original supposition that a positive relationship may exist between blood-sugar level and aggression. A closer inspection of the data suggests the possibility of a threshold in blood-sugar decrease which must be reached before aggression is increased. Thus, it can be seen from Figure 1 that the 4-unit (Group 4) subjects were behaving more like the control (Group $\mathrm{C}$ ) subjects than either the 8- (Group 8) or 12-unit (Group 12) subjects. This would indicate that the threshold may lie somewhere between 4 and 8 units. However, the extreme amount of within-group variability which is typically observed in the shock-elicited aggression situation (see Mollenhour, Voorhees, \& Davis, 1977) may have obscured changes in aggression on the part of the 4-unit subjects.

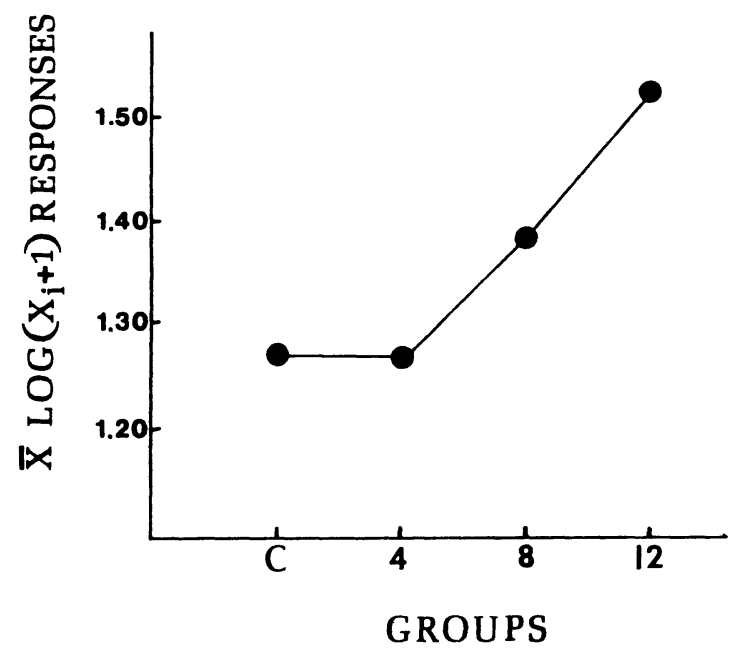

Figure 1. Mean aggressive responses during shock-elicited aggression testing.
Two additional observations, although somewhat speculative, do merit some consideration. First, vocalizations during shock testing, especially on the part of Group 12 subjects (and to a lesser extent on the part of Group 8 subjects), were judged to be considerably more intense that those produced by the other subjects being tested. This suggests that the shock may have been perceived as more painful by these subjects. It was also noted that these intense vocalizations occurred primarily during the initial stages of the test session and dissipated as testing continued. Second, concurrent with the dissipation in vocalization, aggressive responding also tapered off. Thus, it may be that the last few minutes of the testing session did nothing but help obscure differences between groups.

Based upon these results, it is not too difficult to project a number of relevant follow-up studies. For example, manipulation of the shock-session length certainly appears to be warranted. Shortening of the session may well reveal differences that were obscured in the present study, especially in the case of the intermediate (8-unit) group. It would also appear to be of some interest to plot the time course for the development of aggression following insulin injection. As all subjects were tested $1 / 2 \mathrm{~h}$ after injection in the present study, this was not possible.

Also, the comparison of chronic and acute insulin administration is an attractive possibility. It may well be that subjects exposed to lengthy periods of lowered blood sugar prior to shock testing will develop some type of tolerance and not be as aggressive as those subjects experiencing this state for the first time. On the other hand, the converse may prove to be true. And, of course, dosage level itself is a factor that needs further documentation. We would suspect that higher dosage levels would result in a decrement in aggression as debilitating effects (see Wauchope, 1933, for a review of these effects) become paramount. Several of these studies are currently underway.

\section{REFERENCES}

Mollenhour, M. N., Voorhees, J. W., \& Davis, S. F. Sleepy and hostile: The effects of REM sleep deprivation on shockelicited aggression. Animal Learning \& Behavior, 1977, 5, 148-152.

Trotter, R. J. Aggression: A way of life for the Qolla. Science News, 1973, 103, 76-77.

W AUCHOPE, G. M. Critical review hypoglycemia. Quarterly Journal of Medicine, 1933, 5, 117-156.

(Received for publication June 16, 1977.) 